\title{
Prevalência e Fatores Associados à Inatividade Física no Tempo Livre em Adultos na Cidade de Lauro de Freitas-Bahia-Brasil
}

\section{Prevalence and Factors Associated to Physical Inactivity During Leisure Time among Adults from Lauro de Freitas, Bahia, Brazil}

\author{
MARCELA MOTAFREITAS ${ }^{1}$ \\ LUIZALBERTO BASTOS DE ALMEIDA ${ }^{2}$ \\ FRANCISCO JOSÉ GONDIM PITANGA ${ }^{3}$ \\ ESTÉLIO HENRIQUE MARTINS DANTAS ${ }^{4}$
}

\section{RESUMO}

Objetivo: Identificar a prevalência e os fatores sóciodemográficos associados à inatividade física no tempo livre em adultos na cidade de Lauro de Freitas-Bahia-Brasil. Materiais e Métodos: $O$ desenho foi transversal; para cálculo da amostra considerou o tamanho da população de 138.240 habitantes, prevalência da atividade física de $50 \%$, nível de confiança de $95 \%$ de precisão e erro assumido para a prevalência esperada de $5 \%$. Amostra foi constituída de 522 indivíduos com idade e" 18 anos, sendo 220 do sexo masculino e 302 do sexo feminino. O instrumento utilizado foi o International Physical Activity Questionnaire (IPAQ), versão longa além de um Questionário de Identificação para a coleta de informações pessoais. Inicialmente, calculou-se a prevalência da inatividade física no tempo livre, em seguida calculou-se a razão de prevalência por fatores sóciodemográficos. Resultado: Observou-se que a prevalência da inatividade física no tempo livre foi de $82,5 \%$, sendo maior nas mulheres com $87 \%$ em relação aos homens $76,3 \%$. As pessoas com idade $<60$ anos, solteiras, com menor nível de escolaridade e classe social $\mathrm{D}$ apresentaram-se mais inativas. Conclusão: O estudo mostra alta prevalência de inatividade física no tempo livre em adultos na cidade de Lauro de Freitas, assim como, identifica que pessoas com idade menor que 60 anos, solteiras, com baixos niveis de escolaridade e classe social, apresentam associação com a inatividades física no tempo livre. Sugere-se a implantação de programas de incentivo à prática de atividades físicas aos subgrupos populacionais mais afetados, colaborando desta forma com a saúde pública de Lauro de Freitas.

\section{DESCRITORES}

Inatividade física. Tempo Livre. Adultos

\begin{abstract}
Objective: To identify the prevalence and factors associated with physical inactivity during leisure time among adults from Lauro de Freitas, Bahia, Brazil. Material and Methods: This was a cross-sectional study considering a sample population of 138,240 inhabitants, $50 \%$ prevalence of physical activity, $95 \%$ confidence level, and a $5 \%$ error for the expected prevalence. The sample consisted of 522 adults e" 18 years of age, including 220 males and 302 females. The long version of the International Physical Activity Questionnaire (IPAQ) as well as an identification questionnaire to collect personal information were used for data collection. Initially, we calculated the prevalence of physical inactivity during leisure time and then the prevalence ratio for sociodemographic variables. Results: the prevalence of physical inactivity during leisure time was $82.5 \%$, being higher in women $(87 \%)$ as compared to men $(76.3 \%)$. Individuals aged $<60$ years, single, with low education level and those belonging to social class $D$, were found to be more inactive. Conclusion: This study shows a high prevalence of physical inactivity during leisure time among adults from Lauro de Freitas city.

It was further identified that individuals under the age of 60 , single, with low levels of schooling and low-income social class, are associated with physical inactivity during leisure time. It is suggested the implementation of programs to encourage the practice of physical activities to these specific groups, thus collaborating with the community public health in Lauro de Freitas.
\end{abstract}

DESCRIPTORES

physical inactivity, leisure time, adults

Professora do Instituto de Federal de Educação, Ciência e Tecnologia da Bahia - IFBA. Salvador, Bahia.

2 Professor da Universidade Estadual de Feira de Santana (UEFS) - NEPAFS ( Núcleo de Estudo e Pesquisa em Atividade Física e Saúde), Feira de Santana - Bahia

3 Professor da Universidade Federal da Bahia - UFBA. Salvador, Bahia

4 Professor da Universidade Castelo Branco - UCB, Rio de Janeiro. 
$\mathrm{C}$ om o processo da industrialização, existe um crescente número de pessoas que se tornam sedentárias com poucas oportunidades de praticar atividades físicas (AF). O impacto da inatividade física no organismo vem acompanhado de várias doenças e condições metabólicas adversas ${ }^{1}$

Estudos vêm demonstrando que a atividade física regular no tempo livre tem efeito protetor contra doenças crônicas, incluindo hipertensão, doenças da artéria coronária, obesidade, diabete, osteoporose, câncer de cólon, depressão e ansiedade ${ }^{2}$.

Um dos primeiros estudos que avaliou o nível de atividade física no tempo livre (AFTL) foi realizado com 12.138 homens durante 7 anos e, observouse que, AFTL moderada estava associada com $63 \%$ dos eventos fatais e mortes repentinas de doenças crônicas do coração e $70 \%$ de mortes com baixo AFTL. Homens moderadamente ativos tinham taxas de mortalidade $27 \%$ menor do que os sedentários ${ }^{3}$.

Estudos apresentam altos níveis de inatividade física no tempo livre na população europeia ${ }^{4}$ com valores de $67 \%$ na Bélgica e $83 \%$ em Portugal, além de $73 \%$ no Chile 5 . No Brasil, também foram encontrados valores elevados; $80,7 \%$ na cidade de Pelotas ${ }^{6}, 72,5 \%$ em Salvador ${ }^{1}$.

No Brasil, ainda é escassa produ-ção científica que traduza as tendências temporais de $\mathrm{AF}^{7}$. A literatura científica internacional tem mostrado diminuição progressiva dos níveis de atividade física (AF) ocupacionais ${ }^{8}$. Com relação à prática de AF no lazer, há uma aparente estabilização temporal, sendo que em alguns países europeus foi detectado um aumento nos níveis de AF neste domínio nos últimos anos ${ }^{8}$.

Diversos fatores sociodemográficos podem influenciar o comportamento da prática da atividade física em grupos populacionais ${ }^{9}$. O nível socioeconômico, a escolarização, a idade, os aspectos étnicos, entre outras variáveis, vêm sendo relatados na literatura como determinantes da atividade física em seus diferentes domínios ${ }^{10}$. Observa-se que os indivíduos com maior poder aquisitivo e maior escolaridade apresentam-se com maiores chances de se tornarem mais ativos no tempo livre fisicamente ${ }^{11}$.

Em recente trabalho realizado com população de etnia negra da cidade de SalvadorBahia observou-se que indivíduos do sexo masculino, status socioeconômico e nível de escolaridade médio/alto estão inversamente associados com inatividade física no tempo livre (LTPI) em adultos negros ${ }^{12}$.
Portanto, este trabalho teve como objetivo identificar a prevalência e os fatores sócios demográficos associados à inatividade física no tempo livre em adultos na cidade de Lauro de Freitas-Bahia-Brasil.

\section{METODOLOGIA}

Trata-se de estudo de corte transversal realizado no município baiano de Lauro de Freitas situado no nordeste do estado da Bahia, parte da Região Metropolitana de Salvador, com extensão territorial de 59 quilômetros quadrados. O município de Lauro de Freitas possui IDH de 0,771 e PIB per capita R\$12.046,00. De acordo com o Censo Demográfico de 2005 sua população estimada era de 138.240 habitantes $^{13}$.

\section{Amostra}

O cálculo do "n" amostral ${ }^{14}$ levou em consideração os seguintes parâmetros: tamanho da população de 138.240 habitantes, prevalência da atividade física de $50 \%$ por se tratar de média nacional ${ }^{15}$ quando investigada a inatividade física total nos quatro domínios (trabalho, deslocamento, tempo livre e atividade domésticas), bem como da maior prevalência entre as variáveis analisadas no estudo, nível de confiança de $95 \%$ de precisão e erro assumido para a prevalência esperada de $5 \%$.

O tamanho da amostra estimada foi capaz de oferecer, com $95 \%$ de precisão, a prevalência de variáveis de baixa ou elevada freqüência populacional. Para contemplar todos os objetivos desse estudo o " $n$ " estimado foi de 500 adultos $^{3} 18$ anos, ampliado para 600 , de modo a que perdas de qualquer natureza não viessem a interferir na precisão, e que recusas, quando não evitadas, não resultassem em vieses que invalidassem o trabalho.

A amostra foi probabilística, em múltiplos estágios e por conglomerados de classes sociais, informados pela Secretaria de Ação Social da Prefeitura da cidade de Lauro de Freitas, a partir do poder aquisitivo dos moradores dos bairros. Inicialmente, o mapa da cidade foi dividido em microrregiões de acordo com a classe social predominante para visitação dos avaliadores. Tratamos com classe A (alta e média alta), classe $B$ (média), classe $C$ (média baixa) e classe $D$ (baixa e pobreza). Tal procedimento foi adotado para que fossem respeitados todos os níveis de comportamento, acesso a veículos de informação, lazer, transporte, sistema de saúde, educação e 
condição de sobrevivência. A metodologia foi desenvolvida para inclusão de uma amostra com igual número de homens e mulheres.

Em seguida, foram sorteadas probabilisticamente 25 ruas da cidade de Lauro de Freitas pertencentes aos quatro níveis sociais (Classe $A$, $B, C, D$ ) informados pela Secretaria de Ação Social da Prefeitura. A divisão das ruas foi proporcional ao nível sócio-econômico e obedeceu ao seguinte quantitativo: seis ruas em cada um dos conglomerados $\mathrm{A}, \mathrm{B}$ e $\mathrm{C}$ e sete ruas na classe $\mathrm{D}$. Em cada rua, 13 domicílios foram sorteados por amostra sistemática. O intervalo entre as casas variou de acordo com a quantidade de domicílios encontrado em cada rua. A cada residência visitada foram sorteados e entrevistados dois indivíduos adultos (sendo um do sexo masculino e outro do sexo feminino). Foram excluídas pessoas que se recusaram a responder o questionário e pessoas acamadas por problemas de saúde, pois estavam totalmente inativas não por voluntariedade e sim por condição de vida. Também foram excluídas as residências desabitadas; em todos esses casos não houve reposição. Ao final da coleta de dados a amostra ficou constituída de 522 pessoas, 220 do sexo masculino e 302 do sexo feminino.

O presente estudo foi submetido ao Comitê de Ética da Faculdade Adventista de Fisioterapia (FAFIS), localizada na cidade de Cachoeira, estado da Bahia-Brasil, tendo o CAEE: 2363.0.000.070.06 e aprovado através do parecer $n^{\circ}$ 0033/2007. Todos os participantes deste estudo tiveram conhecimento e concordaram previamente com os procedimentos da pesquisa e suas implicações, mediante um termo de consentimento informado.

\section{Coleta de dados}

Cinco avaliadores foram treinados para todas as etapas do trabalho. O índice de confiabilidade inter-avaliadores foi testado através do índice Kappa, para aplicação do IPAQ, e o erro técnico de medidas (ETM), para outras medições ${ }^{16,17}$. Os valores encontrados foram 0,61 e $1,2 \%$, respectivamente, ambos, considerados bons índices de concordância. Todos os participantes da pesquisa foram entrevistados em domicílio. Foi aplicado o questionário IPAQ (International Physical Activity Questionnaire) versão longa, constituído de questões acerca da freqüência e duração da realização de atividades físicas realizadas na última semana desenvolvidas no Trabalho, Deslocamento, Atividades Domésticas e no Tempo livre, porém, para o presente estudo foi trabalhado apenas os valores relacionados ao domínio Tempo livre.

A seguir os valores em METs de cada atividade ${ }^{18}$ realizada para computar e analisar os dados do IPAQ relativos ao domínio Tempo Livre:

Caminhada $=3,3 \mathrm{METs}$, moderada $=4,0$ METs, vigorosa $=8,0 \mathrm{METs}$

Para o cálculo do gasto calórico no tempo livre, multiplicou-se o valor do MET da atividade realizada pela freqüência semanal e duração da mesma; encontrando, então, o gasto calórico no tempo livre em MET minuto/semana. Para transformar em Kcal, multiplicou-se esse valor pelo peso do entrevistado e dividiu-se por 60 minutos ${ }^{18}$. Finalmente, encontrou-se o valor calórico gasto no tempo livre em Kcal durante uma semana.

A partir desse cálculo os indivíduos foram classificados em Ativos ou inativos de acordo com a seguinte referência:

\# Nível de Atividade Física no domínio Tempo livre ${ }^{19}$ :

$$
\text { e" } 1000 \mathrm{Kcal} / \text { semana - ATIVO }
$$$$
<1000 \mathrm{Kcal} / \text { semana - INATIVO; }
$$

No segundo momento, foram coletadas informações pessoais através de Questionário de Identificação, elaborado exclusivamente para esta pesquisa, que consta de dados demográficos como: idade, sexo, estado civil, profissão, grau de instrução e nível social. Para a medida de estatura utilizou-se uma fita métrica antropométrica marca Sanny de $2 \mathrm{~m}$. Com os indivíduos descalços, em posição ereta, com pés e calcanhares unidos $\mathrm{e}$ encostados na parede, braços estendidos ao longo do corpo, respiração normal seguindo o Plano de Frankfurt (linha imaginária que passa pelo ponto mais baixo do bordo inferior da órbita direita e pelo ponto mais alto do bordo superior do meato auditivo externo correspondente; esta linha deve estar paralela ao solo), a fita antropométrica foi posicionada verticalmente ao longo do corpo e com uma régua em posição transversal na altura do vértex mediu-se à distância até região plantar ${ }^{20}$.

$O$ peso foi mensurado através de balança padrão, marca Plenna todas aferidas pelo INMETRO previamente ao estudo (cada uma com certificado próprio) com margem de erro de $\pm 100 \mathrm{~g}$. O entrevistado era solicitado que subisse na balança com os pés descalços, com o mínimo de roupa possível $^{20}$. 


\section{Procedimentos de Análise}

Inicialmente foi realizada a análise descritiva (média, desvio padrão e valores percentuais) da amostra. Na sequência, calculouse a inatividade física no tempo livre da população estudada, e, nos diversos estratos de cada uma das variáveis analisadas (sexo, idade, estado civil, grau de escolaridade, classe social). Depois, calculou-se a inatividade física no tempo livre nos diversos estratos das variáveis analisadas controlando por sexo. Para analisar diferenças entre as variávies contínuas (peso e estatura), foi utilizado o t-test de Student, já, o teste Qui-quadrado, serviu para identificar as diferenças entre os estratos das variáveis categoriais (idade, estado civil, grau de escolaridade e classe social).

Posteriormente, calculou-se a razão de prevalência entre inatividade física no tempo livre e idade, grau de escolaridade, estado civil, classe social. Para esta análise, foram criadas variáveis dummies na comparação entre o grupo de referência $=0$ e os outros estratos de cada variável estudada. As diferenças foram observadas através do teste de Mantel-Hanzel. Utilizou-se o intervalo de confiança a $95 \%$. Nessa fase da análise levouse em consideração o efeito de delineamento amostral (conglomerados) sendo que a ponderação foi feita considerando a unidade amostral domicílios. Os dados foram analisados no programa estatístico STATA 7.0.

\section{RESULTADOS}

As características da amostra são apresentadas na Tabela 1. Observam-se diferenças entre o sexo masculino e feminino na média do peso e estatura.

\begin{tabular}{|c|c|c|c|}
\hline \multicolumn{4}{|c|}{$\begin{array}{l}\text { TABELA 1. Média, desvio padrão e valores percentuais analisados no } \\
\text { estudo. Lauro de Freitas.Bahia. Brasil. } 2007 \text {. }\end{array}$} \\
\hline Variáveis & $\begin{array}{l}\text { Homens } \\
(n=220)\end{array}$ & $\begin{array}{l}\text { Mulheres } \\
(n=302)\end{array}$ & $p$ ou $x^{2}$ \\
\hline Idade (anos) & $\begin{array}{l}40,2 \pm 15,3 \\
\quad(18-85)\end{array}$ & $\begin{array}{c}40,4 \pm 14,7 \\
(20-86)\end{array}$ & 0,880 \\
\hline Peso (Kg) & $\begin{array}{c}75 \pm 14,07 \\
(41-126)\end{array}$ & $\begin{array}{l}68 \pm 14,40 \\
(43,3-129)\end{array}$ & 0,0001 \\
\hline Estatura (m) & $\begin{array}{c}1,70 \pm 0,081 \\
(1,41-1,92)\end{array}$ & $\begin{array}{c}1,58 \pm 0,073 \\
(1,36-1,82)\end{array}$ & 0,0001 \\
\hline \multicolumn{4}{|l|}{ Estado Civil (\%) } \\
\hline Casado & 51,8 & 47,3 & \\
\hline Solteiro & 37,7 & 36,4 & \\
\hline Divorciado & 1,8 & 3,3 & \\
\hline Outros & 8,6 & 12,9 & 0,578 \\
\hline \multicolumn{4}{|l|}{ Grau Escolaridade (\%) } \\
\hline Analf. - Fund. Completo & 44,5 & 45,7 & \\
\hline Médio Incomp.- & 33,6 & 39,4 & \\
\hline Completo & 21,8 & 14,9 & 0,574 \\
\hline \multicolumn{4}{|l|}{$\begin{array}{l}\text { Nível Superior (Incom. - } \\
\text { Pós graduação ) }\end{array}$} \\
\hline \multicolumn{4}{|l|}{ Classe Social (\%) } \\
\hline A & 6,3 & 5,6 & \\
\hline B & 22,7 & 18,8 & \\
\hline C & 66,8 & 68,5 & \\
\hline D & 4,0 & 6,9 & 0,720 \\
\hline
\end{tabular}

\footnotetext{
Valores contínuos foram comparados através do teste "t" de student para amostras independentes e valores percentuais, através do teste qui-quadrado: $x^{2}=$ teste do qui-quadrado.
} 
A prevalência da inatividade física no tempo livre por fatores sócio-demográficos associados na população total do estudo encontra-se na Tabela 2. Demonstra-se alta prevalência de inatividade física no tempo livre $(82,5 \%)$ na população de Lauro de Freitas-Bahia. Entre as variáveis analisadas a maior prevalência encontra-se nas mulheres, em indivíduos com idade $<60$ anos, com menor nível de escolaridade, solteiros e pertencentes à classe social D. Observando diferenças estatisticamente significativas na variável idade, grau escolaridade e classe social.

A prevalência da inatividade física no tempo livre por fatores sócio-demográficos associados estratificados por sexo está apresentada na Tabela 3. Em relação à idade e grau de escolaridade os homens mais inativos no tempo livre encontram-se na faixa etária 40-59 anos e as mulheres com idade até 39 anos. Em ambos os sexos os indivíduos com menor grau de escolaridade são os mais inativos sem diferença estatisticamente significativa entre os diversos estratos dessas variáveis. Quanto ao estado civil, a inatividade no tempo livre predominou entre os homens divorciados e nas mulheres solteiras. Na classe social D observa-se maior nível de inatividade em indivíduos de ambos os sexos.

Na Tabela 4 encontram-se valores referentes à Razão de Prevalência entre inatividade física no tempo livre, idade, sexo, grau de escolaridade, estado civil e classe social.

Os homens têm maior probabilidade de

TABELA 2. Prevalência da inatividade física no tempo livre por fatores sócio-demográficos associados. Lauro de Freitas, Bahia, Brasil, 2007.

INATIVIDADE FÍSICA NO TEMPO LIVRE (\%)

\begin{tabular}{|c|c|c|c|c|c|c|c|}
\hline \multirow[t]{2}{*}{ Variáveis } & \multicolumn{3}{|c|}{ Inativos } & \multicolumn{3}{|c|}{ Ativos } & \multirow[t]{2}{*}{$x^{2}$} \\
\hline & $\mathbf{N}$ & $\%$ & IC95\% & $\mathbf{n}$ & $\%$ & IC95\% & \\
\hline \multicolumn{8}{|l|}{ Sexo } \\
\hline Homens & 168 & 76,3 & $(72,7-79,9)$ & 52 & 23,6 & $(20,0-27,2)$ & \\
\hline $\begin{array}{c}\text { Mulheres } \\
\text { Idade ( anos) }\end{array}$ & 263 & 87,0 & $(84,0-89,9)$ & 39 & 12,9 & $(10,0-15,8)$ & 0,06 \\
\hline $18-39$ & 223 & 83,2 & $(80,0-86,4)$ & 45 & 16,7 & $(15,5-19,9)$ & \\
\hline $40-59$ & 158 & 83,1 & $(79,9-86,3)$ & 32 & 16,8 & $(13,6-20,0)$ & \\
\hline$=60$ & 50 & 78.1 & $(74,6-81,0)$ & 14 & 21,8 & $(18,3-25,3)$ & 0,02 \\
\hline \multicolumn{8}{|l|}{ Estado civil } \\
\hline Casado & 207 & 80,5 & $(77,1-83,9)$ & 50 & 19,4 & $(16,0-22,8)$ & \\
\hline Solteiro & 164 & 84,9 & $(81,9-87,9)$ & 29 & 15,0 & $(12,0-18,0)$ & \\
\hline Divorciado & 11 & 78,5 & $(75,0-82,0)$ & 3 & 21,4 & $(17,9-24,9)$ & \\
\hline Outros & 49 & 84,4 & $(81,3-87,5)$ & 9 & 15,5 & $(12,4-18,6)$ & 0,58 \\
\hline \multicolumn{8}{|l|}{ Grau Escolaridade (\%) } \\
\hline Analf. - Fund. Completo & 210 & 88.9 & $(86,2-91,5)$ & 26 & 11,0 & $(10,6-18,8)$ & \\
\hline Médio Incomp.-Completo & 162 & 83,9 & $(80,8-87,0)$ & 31 & 16,0 & $(13,5-19,7)$ & \\
\hline $\begin{array}{l}\text { Nível Superior (Incom. - Pós } \\
\text { graduação ) }\end{array}$ & 59 & 63,4 & $(59,3-67,5)$ & 34 & 36,0 & $(31,9-40,1)$ & $\begin{array}{l}0,00 \\
1\end{array}$ \\
\hline \multicolumn{8}{|l|}{ Classe social } \\
\hline A & 17 & 54,8 & $(50,6-59,0)$ & 14 & 45,1 & $(40,9-49,3)$ & \\
\hline B & 72 & 67,2 & $(62,5-71,9)$ & 35 & 32,7 & $(28,7-36,7)$ & \\
\hline C & 314 & 88,7 & $(86,0-91,4)$ & 40 & 11,3 & $(8,60-14,0)$ & \\
\hline D & 28 & 93,3 & $(91,2-95,4)$ & 2 & 6,6 & $(4,50-8,70)$ & 0,00 \\
\hline TOTAL & 431 & 82.57 & $(79,2-85,7)$ & 91 & 17.43 & $(14,3-20,7)$ & \\
\hline
\end{tabular}

$\mathrm{x}^{2}=$ teste qui-quadrado. IC95\%-Intervalo de Confiança 
serem inativos na faixa etária 40-59 anos, se forem divorciados (apesar da amostra reduzida), com menor grau de escolaridade e pertencerem às menores classes sociais.

Enquanto que as mulheres se forem solteiras, com menor grau de escolaridade e pertencerem às classes sociais mais baixas têm mais risco de se tornarem inativas fisicamente no tempo livre. Aquelas que são divorciadas apresentam fator de proteção à inatividade física.

\section{DISCUSSÃO}

O presente estudo demonstra alta prevalência de inatividade física no tempo livre
$(82,5 \% ; 79,2-85,7)$ na população de Lauro de Freitas-Bahia. A população européia apresentou $57 \%$ de inatividade física no tempo livre ${ }^{4}$. Dos 16 países europeus pesquisados apenas Portugal, com $83 \%$, apresenta um valor maior no nível de inatividade no tempo livre se comparando com o encontrado no presente estudo. Os outros países apresentaram valores menores como $32 \%$ na Suécia, 33\% na Finlândia, 56\% na Alemanha, 63\% França e $67 \%$ na Bélgica; além de Lodz (Polônia) ${ }^{21}$ $61,1 \%$. No Sudeste e Nordeste do Brasil ${ }^{22}$ a prevalência é de $87 \%$, enquanto que valores menores ${ }^{1,6,23}$ foram encontrados em outros estudos.

Nas 26 capitais do País e no Distrito Federal utilizando dados coletados pelo sistema de

\begin{tabular}{|c|c|c|c|c|c|c|c|}
\hline \multirow[t]{2}{*}{ Variáveis } & \multicolumn{3}{|c|}{ Masculino } & \multicolumn{3}{|c|}{ Feminino } & \multirow[t]{2}{*}{$x^{2}$} \\
\hline & $\mathbf{n}$ & $\%$ & IC95\% & $\mathbf{n}$ & $\%$ & IC95\% & \\
\hline \multicolumn{8}{|l|}{ Idade ( anos) } \\
\hline 18-39 & 87 & 75 & $(71,3-78,7)$ & 136 & 89,4 & $(86,8-92,0)$ & \\
\hline $40-59$ & 60 & 80 & $(76,6-83,4)$ & 98 & 85,2 & $(82,2-88,2)$ & 0,88 \\
\hline$=60$ & 21 & 72,4 & $(68,6-76,2)$ & 29 & 82,8 & $(79,6-86,0)$ & \\
\hline \multicolumn{8}{|l|}{ Estado Civil } \\
\hline Casado & 85 & 74,5 & $(70,8-88,7)$ & 122 & 85,3 & $(82,3-88,3)$ & \\
\hline Solteiro & 63 & 75,9 & $(76,6-79,5)$ & 101 & 91,8 & $(89,5-94,3)$ & \\
\hline Divorciado & 4 & 100,0 & $(0,00-0,00)$ & 7 & 70,0 & $(66,1-73,9)$ & \\
\hline Outros & 16 & 84,2 & $(81,1-87,3)$ & 33 & 84,6 & $(81,5-87,7)$ & 0,05 \\
\hline \multicolumn{8}{|l|}{ Grau Escolaridade (\%) } \\
\hline Analf. - Fund. Completo & 84 & 85,7 & $(82,7-88,7)$ & 126 & 91,3 & $(88,9-93,7)$ & \\
\hline Médio Incomp.-Completo & 59 & 79,7 & $(76,4-83,1)$ & 103 & 86,5 & $(83,6-89,4)$ & \\
\hline $\begin{array}{c}\text { Nível Superior (Incom.-Pós } \\
\text { graduação ) }\end{array}$ & 25 & 52,0 & $(47,8-56,2)$ & 34 & 75,5 & $(71,8-79,2)$ & 0,38 \\
\hline \multicolumn{8}{|l|}{ Classe Social } \\
\hline$A$ & 5 & 35,7 & $(31,6-19,8)$ & 12 & 70,5 & $(66,6-74,4)$ & \\
\hline B & 28 & 56,0 & $(51,8-60,2)$ & 44 & 77,1 & $(73,5-80,7)$ & \\
\hline C & 127 & 86,3 & $(83,3-89,3)$ & 187 & 90,3 & $(87,8-92,8)$ & \\
\hline D & 8 & 88,8 & $(86,1-91,5)$ & 20 & 95,2 & $(93,4-97,0)$ & 0,04 \\
\hline TOTAL & 168 & 76,3 & $(72,6-79,9)$ & 263 & 87,0 & $(84,1-89,8)$ & \\
\hline
\end{tabular}




\begin{tabular}{|c|c|c|c|c|}
\hline Variáveis & $\begin{array}{l}\begin{array}{c}\text { Ambos os } \\
\text { sexos }\end{array} \\
\text { RP (IC95\%) }\end{array}$ & $\begin{array}{l}\text { Masculino } \\
\text { RP(IC95\%) }\end{array}$ & $\begin{array}{l}\text { Feminino } \\
\text { RP (IC95\%) }\end{array}$ & $\begin{array}{c}\mathbf{p} \\
\text { valor }\end{array}$ \\
\hline $\begin{array}{c}\text { Idade ( anos ) } \\
\begin{array}{c}18-39 \\
40-59 \\
=60\end{array}\end{array}$ & $\begin{array}{c}1.00 \\
1.05(0.99-1.13) \\
0.96(0.87-1.06)\end{array}$ & $\begin{array}{c}1.00 \\
1.17(1.03-1.34) \\
1.07(0.90-1.28)\end{array}$ & $\begin{array}{c}1.00 \\
0.96(0.90-1.03) \\
0.90(0.80-1.00)\end{array}$ & $\begin{array}{r}0,001 \\
0,08\end{array}$ \\
\hline $\begin{array}{c}\text { Estado Civil (\%) } \\
\text { Casado } \\
\text { Solteiro } \\
\text { Divorciado } \\
\text { Outros }\end{array}$ & $\begin{array}{c}1.00 \\
1.11(1.05-1.18) \\
0.95(0.80-1.13) \\
1.10(1.01-1.19)\end{array}$ & $\begin{array}{c}1.00 \\
1.05(0.92-1.18) \\
1.51(1.39-1.64) \\
1.24(1.07-1.44)\end{array}$ & $\begin{array}{c}1.00 \\
1.13(1.06-1.20) \\
0.65(0.48-0.89) \\
1.01(0.91-1.11)\end{array}$ & $\begin{array}{l}0,25 \\
0,00 \\
0,02\end{array}$ \\
\hline $\begin{array}{l}\text { Grau Escolaridade (\%) } \\
\text { Nível Superior (Incom. - } \\
\text { Pós graduação } \\
\text { Médio Incomp.-Completo } \\
\text { Analf. - Fund. Completo }\end{array}$ & $\begin{array}{c}1.00 \\
1.23(1.12-1.36) \\
1.39(1.27-1.52)\end{array}$ & $\begin{array}{c}1.00 \\
1.40(1.19-1.65) \\
1.49(1.27-1.75)\end{array}$ & $\begin{array}{c}1.00 \\
1.09(0.97-1.22) \\
1.25(1.13-1.38)\end{array}$ & $\begin{array}{l}0,00 \\
0,05\end{array}$ \\
\hline $\begin{array}{c}\text { Classe Social (\%) } \\
\text { A } \\
\text { B } \\
\text { C } \\
\text { D }\end{array}$ & $\begin{array}{c}1.00 \\
1.21(1.13-1.30) \\
1.37(1.27-1.47) \\
1.70(1.35-2.13)\end{array}$ & $\begin{array}{c}1.00 \\
1.38(1.21-1.56) \\
1.53(1.32-1.76) \\
2.48(1.50-4.12)\end{array}$ & $\begin{array}{c}1.00 \\
1.10(1.01-1.19) \\
1.24(1.15-1.34) \\
1.34(1.08-1.68)\end{array}$ & $\begin{array}{l}0,11 \\
0,01 \\
0,01\end{array}$ \\
\hline
\end{tabular}

IC95\%-Intervalo de Confianç. RP-Razão de Prevalência

Vigilância de Fatores de Risco e Proteção para Doenças Crônicas por Inquérito Telefônico (VIGITEL), implantado em 2006 pelo Ministério da Saúde observou-se que prevalência de inativos no lazer foi superior a $60 \% \mathrm{em} \mathrm{dez}$ cidades. Em Teresina encontrou-se maior prevalência com $68,2 \%$ enquanto Florianópolis apresentou apenas 47,4\% da população inativa fisicamente no lazer ${ }^{24}$. Valores menores do que foi encontrado no presente estudo

As mulheres apresentaram maior prevalência de inatividade física no tempo livre (87\%; $84,0-89,9)$ do que os homens $(76,3 \% ; 72,7-79,9)$ corroborando com vários estudos 9,23,25,26. As diferenças de gênero podem ser explicadas pelos papéis sociais e familiares diferenciados que homens e mulheres adultos exercem, influenciados pela cultura e organização social em que vivem. As mulheres usualmente são as responsáveis pelas tarefas domésticas e cuidadoras de crianças e parentes com limitações, frequentemente também exercem trabalho remunerado ou são dependentes do marido, e acabam tendo menor disponibilidade de tempo e de oportunidades para atividades de lazer ${ }^{27}$.

Com relação à idade o maior nível de inatividade física no tempo livre da população estudada encontra-se nos indivíduos $<60$ anos. Essa associação também foi encontrada na literatura ${ }^{28}$, porém, há estudo que ${ }^{1}$ demonstra uma relação positiva entre a inatividade física no tempo livre e idade.

Dados do VIGITEL 2015 corroboram com essa relação positiva à medida em que demostram que indivíduos com idade entre 18 e 24 anos, 25 e 34 anos, 35 a 44 anos, 45 a 54 anos, 55 a 64 anos e acima de 65 anos apresentam, respectivamente, níveis de atividade física descrescente no lazer, sendo $51,4 \%, 45,2 \%, 36,4 \%, 30,5 \%, 29,1 \%$ e $23,5 \%$ de indivíduos ativos. Ao analisar homens e 
mulheres, separadamente, essa relação também é confirmada ${ }^{29}$.

Trata-se de informação relevante e preocupante. Dados da Organização Mundial de Saúde (OMS) apontam aumento no número de idosos no mundo, isso leva ampliação na desproporcionalidade entre aposentados e jovens produtivos, levando a uma previdência deficitária e com acesso retardado, o que torna fundamental um envelhecimento ativo e produtivo, com a prática regular de atividade física refletindo positivamente no controle de comorbidades inerentes ao envelhecer, como maior conforto para familiares e minimizando a ocupação de horas de leito hospitalar 27.

$\mathrm{Na}$ estratificação por sexo os homens encontram-se mais inativos na faixa etária de 4059 anos $(80 \% ; 76,6-83,4)$ e as mulheres com idade até 39 anos $(89,4 \%: 86,8-92,0)$. Alguns autores ${ }^{1}$ encontraram relação positiva entre inatividade física e idade somente nos indivíduos do sexo masculino; nas mulheres os maiores níveis de atividade física encontram-se em idade a partir de 60 anos. Estudo realizado no estado de São Paulo ${ }^{23}$, a inatividade física no lazer foi menor nas mulheres entre 50 a 59 anos em relação àquelas com menos de 29 anos,

Os resultados sobre a variável idade estratificada por sexo devem sofrer várias influências sócio-econômica e cultural. Na cidade pesquisada, observa-se grande quantidade de casas e centros de assistência às pessoas de meia e terceira idade, onde são desenvolvidas diversas atividades educativas e recreacionais diariamente. A participação da população nessas atividades físicas pode ter contribuído na diminuição do nível de inatividade física encontrado nessa faixa etária.

A explicação para diminuição da inatividade física no tempo livre em mulheres com idade igual ou maior que 60 anos é que nessa, faixa etária, as mulheres já teriam criado seus filhos, muitas vezes já estando aposentadas, tendo, consequentemente, mais oportunidade para participar de atividades físicas no momento de tempo livre 1.

A prevalência da inatividade física no tempo livre foi maior entre os solteiros $(84,9 \% ; 81,9-87,9)$, outro autores encontraram nos indivíduos separados, viúvos e outros $(78,9 \%)^{1}$, e que vivem com companheiro $(82,4 \%)^{6}$.

$\mathrm{Na}$ estratificação por sexo os homens divorciados (100\%), apesar da amostra reduzida, e, mulheres solteiras (91,8\%: 89,5-94,3) demonstraram maior prevalência, porém, alguns estudos ${ }^{1}$ encontraram em homens e mulheres casadas.

Observou-se que os indivíduos com menor grau de escolaridade têm maior nível de inatividade física no tempo livre $(88,9 \% ; 86,2-91,5)$ independente do sexo, vários estudos ${ }^{1,9,6,25}$ têm demonstrado essa associação. Essa tendência se justifica pelo fato de que as pessoas com menor escolaridade têm menos oportunidade de desenvolver atividade física no tempo livre ${ }^{1}$. Apesar de encontrar na literatura estudo ${ }^{30}$ que não encontrou nenhuma associação entre nível de escolaridade e atividade física no tempo livre.

A maior limitação referente a essa pesquisa está relacionada ao estrato do nível social dessa população, à medida que se encontrou extrema dificuldade em ter acesso aos grupos de maior e menor poder aquisitivo. Nos bairros de classe baixa (D) a acessibilidade foi dificultada pelo alto nível de violência, muitas vezes intimidando os entrevistadores devido à falta de segurança. $\mathrm{Na}$ classe mais alta (A), a indisponibilidade dessas pessoas foi marcante, muitas vezes evidenciada pelo medo e receio da presença de um dos entrevistadores em sua residência.

Mesmo diante desse quadro, pode-se observar maior prevalência da inatividade física no tempo livre nos níveis sociais mais baixos $(93,3 \%$; 91,2-95,4) corroborando com os achados de alguns estudos ${ }^{6,9}$. Uma explicação para menor atividade física no tempo livre por pessoas com menor escolaridade seria sua inserção em atividades pesadas e não sedentárias no trabalho, consequentes da baixa qualificação, que é dependente da escolaridade. O inverso ocorre para pessoas com escolaridade mais elevada e atividade sedentária no trabalho ${ }^{1}$. 
O aproveitamento do tempo de lazer com atividades físicas pode levar o indivíduo a se proteger de doenças como hipertensão, diabetes, obesidade. É necessário que os órgãos públicos municipais / estaduais e até privados, além de escolas, tenham iniciativa de desenvolver atividades educativas, além de construção de espaços estruturados e direcionados para a prática de atividade física. Desenvolver programas na área de incentivo a mudança de comportamento com o intuito de informar a importância das atividades físicas no tempo livre em benefício da saúde coletiva.

\section{CONCLUSÃO}

O presente estudo demonstra alto nível de inatividade física no tempo livre da população de
Lauro de Freitas-Ba, sendo que esses dados variam de acordo com os aspectos sócio-demográficos. Baseado nos resultados, pode-se concluir que em relação ao gênero a prevalência da inatividade física no lazer é maior entre as mulheres; assim como em indivíduos com idade $<60$ anos, com menor nível de escolaridade, solteiros e pertencentes à classe social D.

Foi observado que os homens apresentam maior probabilidade de inatividade física no lazer na faixa etária 40-59 anos e, se forem divorciados, com menor grau de escolaridade e pertencerem às menores classes sociais. Quanto às mulheres, as solteiras, com menor grau de escolaridade e classes sociais mais baixas têm mais risco de se tornarem inativas fisicamente no tempo livre.

\section{REFERÊNCIAS}

1. Pitanga F, Lessa I. Prevalência e fatores associados ao sedentarismo no lazer em adultos. Cad. Saúde Pública. 2005; 21(3):870-877.

2. Wagmacker DS, Pitanga FJG. Atividade Física no Tempo Livre como Fator de Proteção para Hipertensão Arterial Sistêmica. R. bras. Ci. e Mov. 2007; 15(1): 69-74.

3. Leon A, Connett J, Jacobs Jr D, Raurama R. Leisure time physical activity levels and risk of coronary heart desease and death: the multiple risk factor intervention trial. JAMA.1987; 258(17):2388-2395.

4. Vuori I. Health benefits of physical activity with special reference to interaction with diet. Public Health Nutr.2001; 4(2B):517-528.

5. Vio D F. Prevención de la Obesidad en Chile. Rev chil nutr. 2005; 32(2): 80-87.

6. Dias-da-Costa J, Hallal P, Wells J, Daltoé T, Fuchs S, Menezes A M et al. Epidemiology of leisure time physical activity: a population-based study in southern Brazil. Cad. Saúde Pública. 2005; 21(1): 275-282.

7. Hallal PC, Knuth AG, Reis RS, Rombaldi AJ, Malta Deborah C, Iser BPM et al . Tendências temporais de atividade física no Brasil (2006-2009). Rev. bras. epidemiol. 2011; 14(Suppl1): 53-60.

8. Knuth AG, Hallal PC. Temporal trends in physical activity: a systematic review. J Phys Act Health. 2009; 6(5):54859.

9. Pitanga FJG, Lessa I, Barbosa PJB, Barbosa SJO, Costa MC, Lopes AS. Fatores sócio-demográficos associados aos diferentes domínios da atividade física em adultos de etnia negra Rev. bras. epidemiol.2012; 15(2):363375.

10. Marquez DX, Neighbors CJ, Bustamante EE. Leisure Time and Occupational Physical Activity among Racial/ Ethnic Minorities. Med Sci Sports Exerc. 2010; 42(6):08693. physically active each day. How can we know? Journal of Nutrition.2001; 131(2):451S-460S.

12. Pitanga FJG, Lessa I, Barbosa PJB, Barbosa SJO, Costa, MC, Lopes AS. Factors associated with leisure time physical inactivity in Black individuals: Hierarchical model. Peer J. 2014;2:e577

13. DATASUS. Dados censitários dos municípios brasileiros. Lauro de Freitas: Ministério da Saúde. http:// www.datasus.gov.br. 2005.

14. Kish L. Survey sampling. New York: John Wiley and Sons; 1965.

15. Hallal P, Victora C, Wells J, Lima R. Physical Inactivity: prevalence and associated variables in Brazilian adults. Med Sci Sports Exerc. 2003; 35(11):894 -900.

16. Pereira M. Epidemiologia: teoria e prática. Rio de Janeiro: Editora Guanabara Koogan;1995.

17. Norton K, Olds T. Antropometrica. Argentina: Biosystem; 2000.

18. Heymsfield S, Lohman T, Wang Z, Going S. Human body composition. Champaign: Human Kinetics, 2005.

19. U. S. Department of Health and Human Services. Physical activity and health: a report of the Surgeon General. Atlanta: National Center for Chronic Disease Prevention and Health Promotion.1996.

20. Pitanga F. Teste, medidas e avaliação em educação física. São Paulo: Editora Phorte, 2004.

21. Kaleta D, Makowiec-dabrowska T, Jegier A Occupational and leisure time energy expenditure and body mass índex. International Journal of Occupational Medicine and Environmental Health 2007; 20(1):9-16.

22. Monteiro C, Conde W, Matsudo S, Matsudo V, Bonseñor I, Lotufo P. A descriptive epidemiology of leisure-time physical activity in Brazil 1996-1997. Rev Panam Salud Publica. 2003; 14(4):246-254. 
23. Zanchetta LM, Barros MBA, César CLG, Carandina L, Goldbaum M, Alves MCGP. Inatividade física e fatores associados em adultos, São Paulo, Brasil. Rev. Bras Epidemiol. 2010; 13(3)387-99.

24. Florindo AA, Hallal PC, Moura EC, Malta DC. Prática de atividades físicas e fatores associados em adultos, Brasil, 2006. Rev. Saúde Pública. 2009; 43(Supl2):6573.

25. Pitanga FJG, Beck CC, Pitanga CPS, Freitas MM, Almeida LAB. Prevalência e fatores sócio demográficos / ambientais associados à atividade física no tempo livre e no deslocamento em adultos. Revista Motricidade. 2014; 10(1):3-13.

26. Sá SSP, Sandre-Pereira G, Salles-Costa R. Fatores sociodemográficos e atividade física de lazer entre homens e mulheres de Duque de Caxias/RJ. Ciênc. saúde coletiva. 2011; 16(11): 4491-4501.

27. WHO - World Health Organization. Gender, health and aging. WHO; 2003. Disponível em http://www.who.int/ gender/documents/en/Gender_Ageing.pdf

28. Salles-Costa R, Heilborn M, Werneck G, Faerstein E, Lopes C. Gênero e prática de atividade física em lazer. Cad Saúde Pública 2003(b);19:S325-S333.
29. Brasil. Ministério da Saúde. Secretaria de Vigilância em Saúde. Departamento de Vigilância de Doenças e Agravos não Transmissíveis e Promoção da Saúde. Vigitel Brasil 2015: vigilância de fatores de risco e proteção para doenças crônicas por inquérito telefônico. Brasília: Ministério da Saúde, 2016.

30. Wolin KY, Bennett GG. Interrelations of Socioeconomic Position and Occupational and Leisure-Time Physical Activity in the National Health and Nutrition Examination Survey. Journal of physical activity \& health. 2008; 5(2):229-241.

\section{Correspondência}

MARCELAMOTAFREITAS

Rua Dr. Rômulo Serrano, apt 207, n 173

Bairro Engenho Velho da Federação- Salvador -Bahia Cep- 40220-005 